\title{
Genetic variability of the pattern of night melatonin blood levels in relation to coat changes development in rabbits
}

\author{
Daniel Allain ${ }^{\mathrm{a} *}$, Benoit Malpaux ${ }^{\mathrm{b}}$, François Puechal ${ }^{\mathrm{a}}$, \\ René Gérard ThÉBAult ${ }^{\mathrm{c}}$, Hubert de Rochambeau ${ }^{\mathrm{a}}$, \\ Philippe CHEMINEAU ${ }^{\mathrm{b}}$ \\ a Station d'amélioration génétique des animaux, Institut national de la recherche agronomique, \\ BP 27, 31326 Castanet Tolosan, France \\ ${ }^{\mathrm{b}}$ Physiologie de la reproduction et des comportements, neuroendocrinologie, Institut national \\ de la recherche agronomique, 37380 Nouzilly, France \\ ${ }^{\mathrm{c}}$ Génétique animale phanères, Le Magneraud, Institut national de la recherche agronomique, \\ BP 56, 17700 Surgères, France
}

(Received 6 June 2002; accepted 25 September 2003)

\begin{abstract}
To assess the genetic variability in both the nocturnal increase pattern of melatonin concentration and photoresponsiveness in coat changes, an experiment on 422 Rex rabbits (from 23 males) raised under a constant light programme from birth was performed. The animals were sampled at 12 weeks of age, according to 4 periods over a year. Blood samples were taken 7 times during the dark phase and up to $1 \mathrm{~h}$ after the lighting began. Maturity of the fur was assessed at pelting. Heritability estimates of blood melatonin concentration $(0.42,0.17$ and 0.11 at mid-night, 13 and $15 \mathrm{~h}$ after lights-out respectively) and strong genetic correlations between fur maturity and melatonin levels at the end of the dark phase $(-0.64)$ indicates that (i) the variability of the nocturnal pattern of melatonin levels is under genetic control and (ii) the duration of the nocturnal melatonin increase is a genetic component of photoresponsiveness in coat changes.
\end{abstract}

rabbit / coat development / melatonin secretion / genetic variability

\section{INTRODUCTION}

The pineal gland, via melatonin secretion is a key element in the neuroendocrine control of pelage changes by photoperiodism in various mammalian species including mink, sheep, goats and rabbits [1] as it is for many other functions including reproduction, the regulation of body weight, the immune

${ }^{*}$ Corresponding author: allain@ $@$ toulouse.inra.fr 
system, thyroid activity, thermoregulation, the control of neoplastic growth and more generally the control of circadian rhythms [2]. The photoperiodic information received by the retina is transduced into a neuroendocrine signal through the nocturnal duration of the increase in the pineal hormone, melatonin, [7]. It is well established that nocturnal increases of blood melatonin levels are highly variable in both duration and amplitude amongst individuals. Recently, it has been shown in sheep that the variability in night-time melatonin plasma levels is under a strong genetic control [13] but no similar information is available about the duration of the nocturnal increase of melatonin levels. Furthermore, it is well known that the time of coat changes in response to changes in the photoperiod are variable among individuals and could be modified by a selection programme in some mammals [6].

The study presented in this paper was conducted to assess genetic variability in both the nocturnal pattern of melatonin plasma concentrations and coat changes in the rex rabbit bred for fur production.

\section{MATERIALS AND METHODS}

\subsection{Animals}

This experiment was carried out at the fur rabbit farm of the Institut national de la recherche agronomique, Le Magneraud, France. Rabbits were born between January and mid-September 1998. The animals were maintained under long photoperiods (LD 16:8, light from 4 to $20 \mathrm{~h}$ ) from birth to 8 weeks of age and then moved to short photoperiods (LD 8:16, light from 8 to $16 \mathrm{~h}$ ) until the blood sampling procedure which occurred at 12 weeks of age corresponding to the onset of coat replacement induced by light treatment [11]. The rabbit colony is managed in 10 reproduction groups composed of 1 male and 10 females with overlapping generations. Within each group a sire is always replaced by one of his sons while female replacement is made with animals born in other paternal groups. The animals were issued from a line of Rex rabbits selected for several fur production traits: live bodyweight at 8 weeks and hair length, fur compactness, bristliness score and fur maturity score observed at 16 weeks of age [9]. A total of 422 animals of both sexes born from 23 sires (50 to 55 animals/reproduction group; 3 to 40 animals/sire) were used and their genealogy was known over about ten generations so that a total of 24473 animals were included in the pedigree file. The animals were housed individually from 8 weeks of age in closed buildings with a natural ventilation and where the temperature varied between $15^{\circ} \mathrm{C}$ in the Winter and $25^{\circ} \mathrm{C}$ in the Summer. 
The animals were fed ad libitum with a complete pelleted commercial food and had free access to water.

\subsection{Blood sampling}

The animals were blood sampled at 12 weeks of age according to four different periods over the year 1998: mid-March, mid-June, mid-September or mid-December which correspond to solstice and equinox times. At each period, two groups of 50 animals were sampled at a weekly interval as allowed by time; a maximum of 50 rabbits could be sampled within one hour. For each rabbit, blood sampling occurred 6 times during the dark phase at the 5th, 6th, 7th, 8th, 13th and 15th hour after the lights were switched off and one time during the light phase $1 \mathrm{~h}$ after the lights went on. This blood sampling design during the night was used in order to determine the amplitude and the duration of the nocturnal increase of melatonin secretion. The amplitude was evaluated by meaning plasma melatonin concentrations from the fifth to the eighth hour of the dark phase. The two blood samples taken in the late dark phase were indicators of the duration since in most species including the rabbit [12], melatonin secretion begins as soon as the lights are switched off. Blood samples were obtained by venipuncture of the ear artery and were collected under dim red light $(<1$ lux at $20 \mathrm{~cm}$ ), avoiding any direct illumination of the eyes. Plasma was immediately separated by centrifugation and stored at $-20{ }^{\circ} \mathrm{C}$ until assay.

\subsection{Radioimmunoassays}

Plasma melatonin concentrations were estimated from a $250 \mu \mathrm{L}$ aliquot of blood plasma. An extraction with $2 \mathrm{~mL}$ of chloroform was first performed. After freezing at $-20{ }^{\circ} \mathrm{C}$ and total evaporation of the organic phase, the extract was diluted in $700 \mu \mathrm{L}$ of a tricine buffered saline, then two aliquots of $300 \mu \mathrm{L}$ were taken for radioimmunoassay according to the technique of Fraser et al. [4] with an antibody raised by Tillet et al. [10]. The sensitivity of the assay was $4 \mathrm{pg} \cdot \mathrm{mL}^{-1}$.

\subsection{Measurement of fur priming on dry pelts}

At pelting (18 weeks of age), the skins were fleshed and dried. A picture from the leather side with a digital camera was taken and the priming of the fur was evaluated by measuring the area of pigmented blue skin with the help 
of a specific routine developed under the Optimas ${ }^{\circledR}$ imaging analysis software. The skin is blue pigmented during the active phase of hair follicle growth due to both an intense vascularisation around the hair bulb and incorporation of melanin within the hair, while during the rest phase and due to the absence of cell multiplication, the skin is unpigmented and nearly white. The rate of fur priming was determined by the ratio of the area of blue pigmented skin to total skin area.

\subsection{Statistical analysis}

A mean mid-night melatonin plasma concentration was assessed for each rabbit from the four samples taken during mid-night at hourly intervals from the 5th to 8th hours after lights off. Normalisation of the variable rate of fur priming area, ranging from 0 to 1 , was performed using an arcsine transformation. Genetic parameters for all traits $(\mathrm{Y})$ were estimated in a single run using REML VCE, a multivariate restricted maximum likelihood variance component estimation programme $[5,8]$ with an animal model according to the following mixed linear model:

$$
\boldsymbol{Y}_{i j k l m}=\mu+S_{i}+\boldsymbol{M}_{\boldsymbol{j}}+\boldsymbol{B}_{\boldsymbol{k}_{\boldsymbol{j}}}+\boldsymbol{a}_{l}+\boldsymbol{c}_{m}+\boldsymbol{e}_{i j k l m}
$$

where $\boldsymbol{Y}_{i j k m}$ is a matrix of traits measured on animal $\boldsymbol{l}, \boldsymbol{\mu}$ is the overall mean, $\boldsymbol{S}_{i}$ is the sex effect (two levels: male or female), $\boldsymbol{M}_{j}$ is the period of the year effect (four levels: March, June, September or December), $\boldsymbol{B} \boldsymbol{G}_{j k}$ is the bleeding group within the period of the year effect (eight levels: two bleeding groups per period), $\boldsymbol{a}_{l}$ is the random vector of additive genetic effects of animals, $\boldsymbol{c}_{m}$ is the random vector of common environmental effects for all animals from the same litter, and $\boldsymbol{e}_{i j k l m}$ is the random vector of residuals. The additive genetic, litter and residual vectors were assumed as normally distributed random effects, a $\mathrm{N}\left(0, \mathrm{~A} \sigma^{2} \mathrm{a}\right), \mathrm{c} \sim \mathrm{N}\left(0, \mathrm{I} \sigma^{2} \mathrm{c}\right), \mathrm{e} \sim \mathrm{N}\left(0, \mathrm{I} \sigma^{2} \mathrm{e}\right)$ where $\mathrm{A}$ is the relationship matrix among animals, $\mathrm{I}$ is the identity matrix, and $\sigma^{2} \mathrm{a}, \sigma^{2} \mathrm{c}$ and $\sigma^{2} \mathrm{e}$ are the additive genetic, litter and residual variances respectively.

The phenotypic variance was estimated by the sum of the estimated additive genetic, common environment and residual variance. Heritability was calculated as the ratio of estimated additive genetic variance on phenotypic variance.

\section{RESULTS}

Variations of mean plasma melatonin concentrations during the night are reported in Table I. High concentrations (between $154.3 \pm 3.4$ to 
Table I. Descriptive statistics of plasma melatonin concentrations at different times during the night and the rate of fur priming area at pelting.

\begin{tabular}{lccc}
\hline \multicolumn{1}{c}{ Traits } & N & Mean & $\begin{array}{c}\text { Coefficient } \\
\text { of variation (\%) }\end{array}$ \\
\hline Melatonin concentrations $\left(\mathrm{pg} \cdot \mathrm{mL}^{-1}\right)$ & & & \\
D5 (5 h after lights out) & 421 & 174.7 & 43.2 \\
D6 (6 h after lights out) & 419 & 171.3 & 45.9 \\
D7 (7 h after lights out) & 420 & 161.4 & 44.7 \\
D8 (8 h after lights out) & 421 & 154.3 & 45.8 \\
Mean mid-night (D5 to D8) & 419 & 165.5 & 38.6 \\
D13 (13 h after lights out) & 421 & 143.9 & 47.7 \\
D15 (15 h after lights out) & 421 & 36.1 & 141.8 \\
L1 (1 h after lights went on) & 412 & 7.3 & 63.0 \\
Rate of fur priming area (\%) & 255 & 0.82 & 19.5 \\
\hline
\end{tabular}

$174.7 \pm 3.7 \mathrm{pg} \cdot \mathrm{mL}^{-1}$ ) were observed around mid-night from the fifth to the eighth hour of the dark phase. Mean mid-night plasma concentrations of melatonin were $165.5 \pm 3.1 \mathrm{pg} \cdot \mathrm{mL}^{-1}$. Plasma melatonin concentrations remained high $13 \mathrm{~h}$ after lights out $\left(143.9 \pm 3.3 \mathrm{pg} \cdot \mathrm{mL}^{-1}\right)$. Thereafter a sharp decrease was observed. Fifteen hours after lights out, the mean plasma melatonin level was $36.1 \pm 2.5 \mathrm{pg} \cdot \mathrm{mL}^{-1}$ and close to zero $\left(7.3 \pm 0.2 \mathrm{pg} \cdot \mathrm{mL}^{-1}\right)$ one hour after the onset of the light phase.

A very large variation between the animals was observed in the decrease of melatonin in the late part of the dark phase (Tab. I). Fifteen hours after lights out, some animals exhibited high levels similar to those observed at mid-night while very low levels close to zero and similar to diurnal levels were observed in the others.

Heritabilities, and phenotypic and genetic correlations of plasma melatonin concentrations over the dark phase and rate of fur priming area at pelting are shown in Table II.

Heritability estimates of plasma melatonin concentrations were moderate during the night (from 0.42 at mid-night to 0.11 for D15) and low during the daylight period (0.05). 


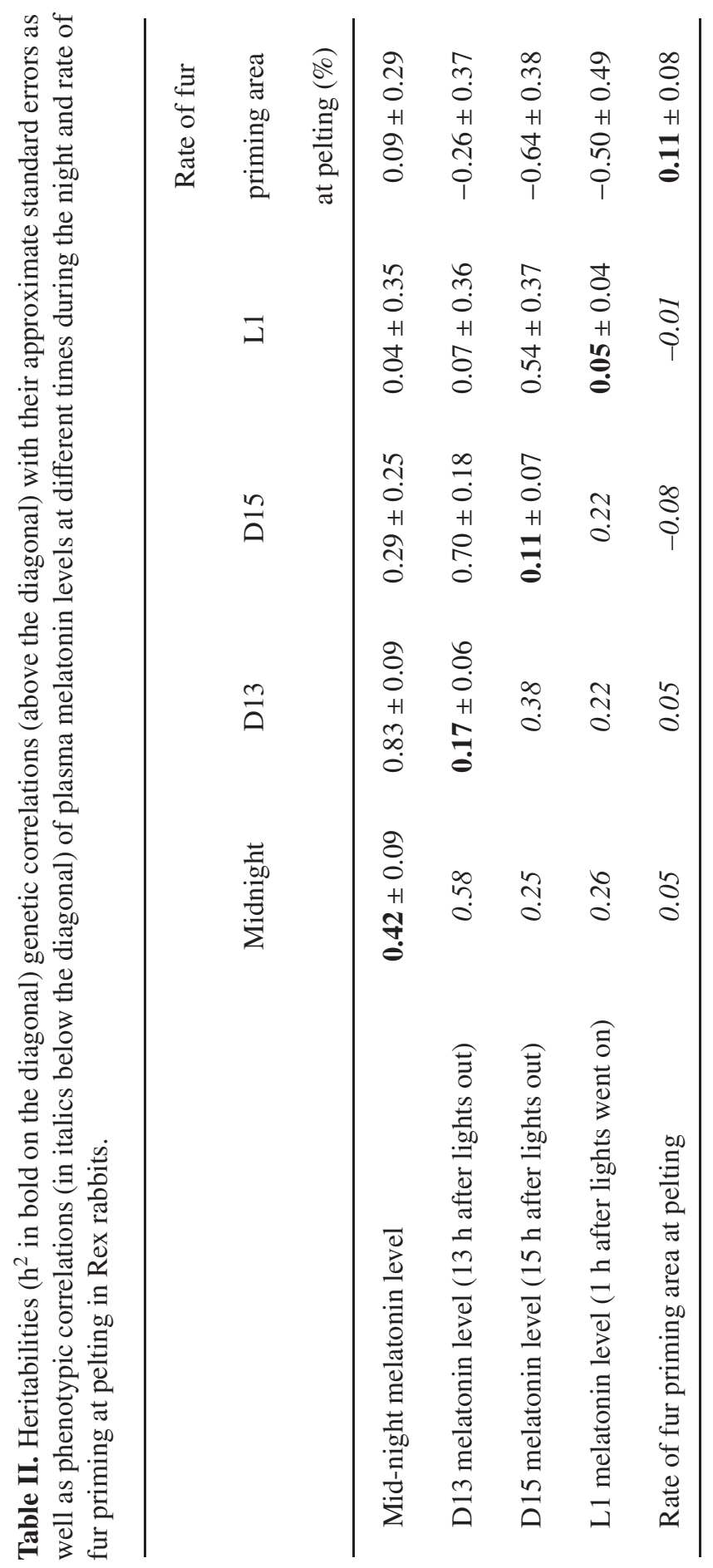


Genetic correlations between melatonin levels in consecutive samples were positive and high ( 0.54 to 0.83 ) but decreased as the interval between sampling time increased.

There were high negative genetic correlations between the rate of fur priming and melatonin levels in the late part of the dark phase or $1 \mathrm{~h}$ after the lights went on and low or close to zero with melatonin levels at mid-night.

\section{DISCUSSION}

The heritability estimate of mid-night plasma melatonin concentration was moderate and similar to that observed in ewes [13]. This proves that the large variability in mean melatonin plasma concentrations at mid-night is under genetic control in rabbits as it is in sheep [3]. Moreover it has also been shown that the genetic variability in melatonin concentrations in ewes originates in its synthesis, and not in its catabolism [14]. We can suppose that it will be similar in rabbits.

The moderate heritability estimates of plasma melatonin concentrations in the late part of the night $(0.17$ and 0.11 at 13 and $15 \mathrm{~h}$ after lights out, respectively) are more interesting. Such parameters are indicators of the duration of the nocturnal increase of melatonin, which, in most species including the rabbit [12], begins as soon as the light is switched off. Thus, the very large phenotypic variability in melatonin concentrations in the late part of the dark phase observed between animals is under genetic control and indicates that some animals are able to anticipate the end of the dark phase. To our knowledge, no other reports about the genetic control of the duration of plasma melatonin levels during the night in any mammalian species has been published. Therefore the duration of the nocturnal increase of plasma melatonin levels transduces the photoperiodic information and is the main cue of seasonal function changes in response to photoperiod changes [7].

Furthermore, the measurement of the rate of fur priming area at pelting is an indicator of the photoresponsiveness of coat changes. The animals were raised under a lighting pattern that induces coat development changes in rabbits [11]. There is no genetic relationship between the rate of the fur priming ratio and melatonin levels at mid-night. But strong and negative genetic correlation estimates were observed with melatonin concentrations in the later part of the dark phase or $1 \mathrm{~h}$ after the lights were switched on, -0.64 and -0.50 , respectively. The lower were the melatonin levels, the higher the rate of fur priming area. Such result would first indicate that the main cue of coat changes photoresponsiveness is the duration of high nocturnal melatonin levels rather than the levels 
at mid-night. This confirmed that photoperiodic information is transduced by the duration of a nocturnal increase of plasma melatonin levels [7]. Secondly, it appears that under a short day photoperiod, the animals exhibiting a shorter duration of nocturnal high melatonin levels would respond better to the photoperiod. Thus the duration of the nocturnal plasma melatonin increase which is under genetic control, could be an interesting genetic component of photoresponsiveness. A selection experiment with divergent lines upon the duration of nocturnal increase of melatonin concentrations and measurement of correlated responses on seasonal functions would confirm our results. Additionally such a divergent selection on nocturnal melatonin levels may be useful to elucidate the physiological mechanisms underlying the regulation of melatonin blood levels in mammals.

The experimental design used in this study was to assess the genetic variability of the two main components of the nocturnal increase of melatonin concentration, i.e. amplitude and duration, in relation to photoresponsiveness in coat changes. Thus blood samples and melatonin concentration measurements were first defined in order to determine the amplitude or the maximum of the nocturnal increase of melatonin secretion and to obtain indicators of the duration of this secretion pattern, but not for precisely describing the whole secretion pattern all over the night. These main components, amplitude and duration of the nocturnal increase of the melatonin concentration, could be interpreted as independent time variables since it has been shown that the variability of melatonin concentration during the dark phase originates in its synthesis rather than in its catabolism since mid-life duration of melatonin in the blood is about $20 \mathrm{~min}$ [14], i.e. ten times shorter than the interval between blood samples at mid-night and those in the late dark phase. Thus due to the first aim of this study and to the rapid catabolism of melatonin in the blood the data were analysed according to a classical linear generalised model rather than a longitudinal data one. However, any autoregressive structure of our data cannot be fully excluded since genetic correlations between consecutive samples decrease as the time interval increases. Thus it would be useful to study more precisely the pattern of melatonin secretion at regular intervals during the night in a future investigation and to analyse data with a model for longitudinal data.

In conclusion, the variability of both the mid-night melatonin blood levels and the duration of the nocturnal increase of melatonin levels are under genetic control in rabbits. Moreover, there is a genetic link between melatonin levels in the late part of the dark phase and photoresponsiveness in coat changes. 


\section{ACKNOWLEDGEMENTS}

The authors thank Claude Cahier and Michel Vigneau of the Institut national de la recherche agronomique (Inra), Physiologie de la reproduction et des comportements, Nouzilly Research Centre, Gérard Auvinet, Michel Bonnet and Jean Claude Musseau of Inra Génétique animale, Le Magneraud and Jacky Falières, Inra Toulouse, for the supply and care of animals and collection of blood samples.

\section{REFERENCES}

[1] Allain D., Thebault R.G., Rougeot J., Martinet L., Biology of fibre growth in mammals producing fine fibre and fur in relation to control by daylength: relationship with other seasonal functions, in: Laker J.P., Allain D. (Eds.), Hormonal control of fibre growth and shedding, European Fine Fibre Network Ocasional Publication no. 2, Macaulay Land Use Research Institute, Aberdeen, 1994, pp. 23-40.

[2] Arendt J., Physiology of the Pineal: role in photoperiodic seasonal functions, in: Arendt J. (Ed.), Melatonin and the Mammalian Pineal Gland, Chapman \& Hall, London, 1995, pp. 110-158.

[3] Chemineau P., Zarazaga L., Malpaux B., Gomez-Brunet A., Guillaume D., Ravault J.P., Bodin L., Coon S., Weller J., Klein D., Voisin P., Variability in nightime melatonin plasma concentration is under strong genetic control in the Ile-de-France sheep, in: Proceedings 6th World Congress Genetics Applied to Livestock Production, 11-16 January 1998, Vol. 27, University of New South Wales, Armidale (Australia), pp. 59-62.

[4] Fraser S., Cowen P., Franklin M., Franey C., Arendt J., Direct radioimmunoassay for melatonin in plasma, Clin. Chem. 20 (1983) 396-397.

[5] Groeneveld E., VCE4. User's guide and reference manual, 1997.

[6] Lynch G.R., Lynch C.B., Kliman R.M., Genetic analyses of photoresponsiveness in the Djungarian hamster, Phodopus sungorus, J. Comp. Physiol. A 164 (1989) 475-481.

[7] Malpaux B., Viguie C., Skinner D.C., Thiery J.C., Pelletier J., Chemineau P., Seasonal breeding in sheep: mechanism of action of melatonin, Anim. Reprod. Sci. 42 (1996) 109-117.

[8] Neumaier A., Groeneveld E., Restricted maximum likelihood of covariances in sparse linear models, Genet. Sel. Evol. 30 (1998) 3-26.

[9] Thebault R.G., Allain D., Rochambeau H. de, Vrillon J.L., Selection scheme and genetic improvement of Orylag for fur production, in: Proceedings of the VIIth International Congress in Fur Animal Production, 13-15 September 2000, Scientifur, Vol. 24, Kastoria (Greece), pp. III 81-86. 
[10] Tillet Y., Ravault J.P., Selve C., Evin G., Castro B., Dubois M.P., Conditions for the use of specific antibodies for immunohistochemical visualization of serotonin and melatonin in the pineal gland of sheep, [in French], C. R. Acad. Sci. Série III, Sciences de la Vie, 303 (1986) 77-82.

[11] Vrillon J.L., Thebault R.G., Rochambeau H. de, Dardant P., Photoperiodism effect on fur maturity and fur quality of rabbits, owning or not rex gene, in: Proceedings of the 4th Congress of the World Rabbit Science Association, 10-14 October 1988, Vol. 2 (Genetics Physiology), Budapest (Hungary), pp. 244-252.

[12] Young Lai E.V., Pang S.F., Brown G.M., Effects of different photoperiods on circulating levels of melatonin and $\mathrm{N}$-acetylserotonin in the female rabbit, Acta Endocrinol. 112 (1986) 145-149.

[13] Zarazaga L.A., Malpaux B., Bodin L., Chemineau P., The large variability in melatonin blood levels in ewes is under strong genetic influence, Amer. J. Physiol. 274 (1998) E607-E610.

[14] Zarazaga L.A., Malpaux B., Guillaume D., Bodin L., Chemineau P., Genetic variability in melatonin concentrations in ewes originates in its synthesis, not in its catabolism, Am. J. Physiol. 274 (1998) E1086-E1090.

To access this journal online: www.edpsciences.org 